\title{
Multidisciplinary family-centred psychosocial care for patients with CHD: consensus recommendations from the AEPC Psychosocial Working Group
}

\author{
Elisabeth M.W.J. Utens, ${ }^{1,2}, *$ Edward Callus, ${ }^{3}{ }^{3} *$ Eveline M. Levert, ${ }^{1}$ Katya De Groote, ${ }^{4}$ Frank Casey ${ }^{5,6} \ddagger$ \\ ${ }^{1}$ Department of Child and Adolescent Psychiatry/Psychology, Erasmus MC-Sophia Children's Hospital, Rotterdam, \\ The Netherlands; ${ }^{2}$ Research Institute of Child Development and Education, University of Amsterdam/de Bascule, \\ Academic Center for Child and Adolescent Psychiatry, Amsterdam, The Netherlands; ${ }^{3}$ Clinical Psychology Service, \\ IRCCS Policlinico San Donato, San Donato Milanese, Milan, Italy; ${ }^{4}$ Department of Paediatrics, Division of Paediatric \\ Cardiology, Ghent University Hospital, Ghent, Belgium; ${ }^{5}$ Department of Paediatric Cardiology, Royal Belfast Hospital \\ for Sick Children; ${ }^{6}$ Department of Child Health, Queen's University, Belfast, Northern Ireland
}

\begin{abstract}
Because of the enormous advances in the medical treatment of $\mathrm{CHD}$, the long-term survival of patients suffering from this disease has increased significantly. Currently, about $90 \%$ of patients reach adulthood, which entails many new challenges both for patients and their families and for healthcare professionals. The main objective of family-centred psychosocial care is to strengthen the emotional resilience of chronically ill patients and their families by adopting a holistic approach. During the biannual meeting of the psychosocial working group in 2012, participants expressed the need for general European guidelines. The present recommendations were written to support medical staff and psychosocial healthcare professionals to provide the best care for children and adolescents with CHD as well as for their families. This article describes in detail how the integrated family-centred psychological care modules work, involving different healthcare specialists, including a paediatric/congenital cardiologist or a general paediatrician. The different clinical implications and specific needs have been taken into account and recommendations have been provided on the following: structured follow-up screening; identification of stressful periods related to cardiac surgery or invasive medical procedures; evidence-based, disease-specific, and familyoriented psychosocial interventions; and interactive media links to medical and psychosocial information.
\end{abstract}

Keywords: CHD; psychosocial; recommendations; children; parents

Received: 9 January 2017; Accepted: 10 June 2017; First published online: 11 September 2017

$\mathrm{B}$ ECAUSE OF THE ENORMOUS ADVANCES IN THE medical treatment of children born with CHD, the long-term survival rate of these children has increased significantly. ${ }^{1}$ Currently, about $90 \%$ of these patients reach adulthood. This increased

Correspondence to: E. Utens, Department of Child and Adolescent Psychiatry/ Psychology, Erasmus MC-Sophia Children's Hospital, Wytemaweg 8, KP-2 building, 3015 CN Rotterdam, The Netherlands. Tel: +31 10 7040209; E-mail: e.utens@erasmusmc.nl

* Elisabeth Utens and Edward Callus share first authorship.

$\$$ The original version of this article was published with the incorrect affiliation information. A notice detailing this has been published and the error rectified in the online and print PDF and HTML copies. survival rate is accompanied by long-term morbidity, which may negatively influence the emotional adjustment of patients and their parents. Children with CHD, unfortunately, suffer from more behavioural, emotional, and neuropsychological problems when compared with their healthy peers. ${ }^{2-4}$ Moreover, research showed that, despite evident improvements in the treatment of CHD over the past decades, no clear improvement was made as to behavioural and emotional problems of these children and their parents. $^{5}$

In 2012, the American Heart Association recommended ${ }^{4}$ screening and identification of children at 
risk for developmental disorders and neuropsychological, emotional, and social problems, in order to provide them with adequate services and prevent the development of additional problems. This statement consists of a "state of the art" review, based on an extensive literature review, and it describes how to implement screening, surveillance, and monitoring in these children. It is recommended to assess and treat psychopathological problems of CHD patients at a young age. Intervening early is also imperative in order to avoid the persistence of psychopathological problems from childhood through adolescence into adulthood. ${ }^{6}$ Moreover, youngsters with CHD and their parents have convincingly expressed their need for psychosocial care in several studies. ${ }^{7,8}$ In addition, psychosocial care is important as medically related trauma and adjustment problems of children and parents can negatively affect the relationship with medical staff and treatment adherence.

Positive outcomes of psychosocial interventions by the Belfast group, targeting well-being and adjustment in infants and schoolchildren with CHD and their mothers, have been already described in the literature. ${ }^{9,10}$ At present, their protocol for schoolchildren is being further studied in a Dutch randomised control trial, with the aim to improve emotional and school functioning in these children. Moreover, standardised exercise training has been shown to improve the quality of life in children with CHD. ${ }^{11}$ When it comes to cognitive/neurognitive development, evidence-based psychosocial interventions to promote intellectual functioning are still lacking and need to be developed.

During the biannual meeting of the AEPC Psychosocial Working Group in 2012, participants expressed the need for specific European recommendations. This paper, therefore, aims to extend previous work by stating briefly the type of psychosocial care that should be provided by a particular healthcare professional during the different developmental phases of the patient. It presents the clinical perspective of the AEPC Psychosocial Working Group on how integrated family-centred psychosocial care should ideally be provided for families with children, adolescents, or adults with CHD.

This group is aware that financial resources are limited, that health insurances do not cover all costs for psychosocial care, and that highly trained health professionals to provide psychosocial care are not yet available in all paediatric and adult congenital cardiology units. It should be stressed that the recommendations presented in this article therefore outline an ideal setting. Even if this standard cannot be achieved by all centres, healthcare providers should continue to strive for optimal psychosocial care. These guidelines are based on extensive clinical experience and scientific evidence ${ }^{3,4,12}$ indicating behavioural, emotional, social, and neuropsychological problems in CHD patients and coping and adjustment problems in their parents.

\section{For whom are these short guidelines intended?}

These guidelines are written for members of the medical staff, medical doctors, paediatric and congenital cardiologists, cardiac surgeons, nurses, nurse practitioners, and psychosocial healthcare professionals such as psychologists, social workers, child-life specialists, and religious professionals working with children, adolescents, or adults with CHD and their families.

\section{Multidisciplinary family-centred psychosocial care}

\section{Aims of multidisciplinary family-centred psychosocial care}

The main objective of family-centred psychosocial care is to strengthen the emotional resilience of chronically ill patients and their families. It aims to enhance a "holistic" treatment of the patient and the family as "a whole" - that is, from a body and mind perspective - and the psychosocial development of the patient and his or her family.

\section{Levels of multidisciplinary family-centred psychosocial care}

General, non-disease-specific psychosocial interventions intend to decrease negative risk factors and strengthen protective factors in a family. ${ }^{10,13}$ Psychosocial care can be provided at three levels:

1. At the level of the individual child with chronic illness, psychosocial counselling or psychotherapeutic treatment is focused on learning how to cope with physical limitations associated with the chronic condition, to cope with negative emotions related to disease or medical procedures - such as anxiety, anger, sadness, despair, post-traumatic stress, and hopelessness or helplessness - and to cope with a loss of control over one's own body and life. $1,5,8,10-12$

2. At the level of parents, counselling or psychotherapeutic treatment is focused on providing support to parents regarding their social issues - such as arranging parental leave for cardiac surgery of the child, unemployment, financial problems emotional issues - such as post-traumatic stress and fostering parenthood and parenting the CHD child. ${ }^{14-16}$

3. At the family level, counselling/psychotherapeutic treatment aims to gain insight into the interrelationships within the family, the impact of the 


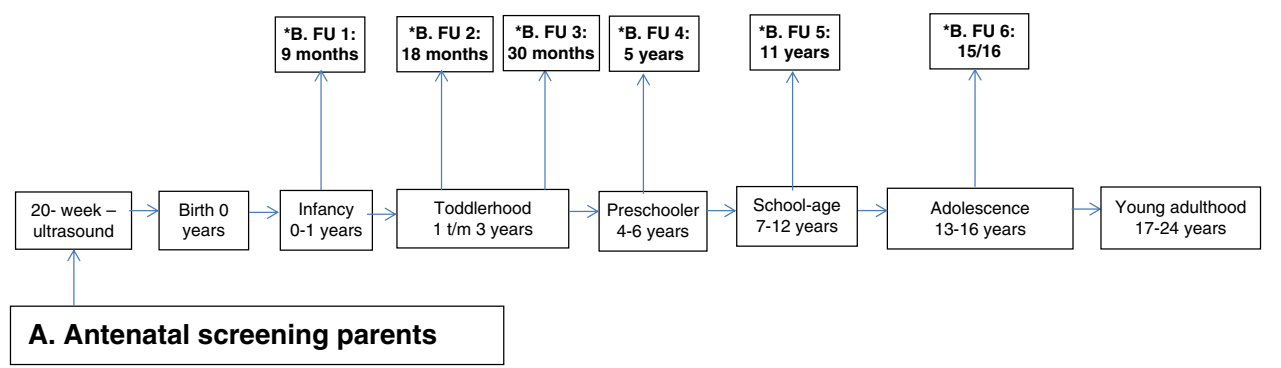

B. Psychological follow-up (see *B: follow-up moments)

C. Medical: outpatient examinations

D. Medical: hospitalizations (cardiac surgery/catheterizations)

E. Transition

F. Death

Figure 1.

Scheme for multidisciplinary family-centred psychosocial care.

chronic illness on each of the family members, and the effect of stress on mutual relationships. It intends to reduce negative interactions and teach adequate problem-solving and coping strategies. As families usually present with several different problems at a time, psychosocial care is probably most effective when it is organised in a multidisciplinary setting. ${ }^{17}$

We recommend integrated family-centred psychosocial care and will describe modules attuned to the different developmental phases of patients with $\mathrm{CHD}$ (see Fig 1).

\section{Healthcare providers involved in psychosocial care}

Psychosocial care can be provided by child-life specialists, social workers, clinical psychologists, specialised neuropsychologists, psychotherapists, psychiatrists, and religious counsellors such as priests, ministers, imams, rabbis, monks, or religious figures pertinent to the patient and family. More specifically for CHD it also includes counselling and/or psychotherapy provided by a licensed psychologist, a child and adolescent psychiatrist, or specialised nurses, with regard to providing procedural information.

Preferably, these professionals should collaborate in a multidisciplinary team with medical specialists such as paediatricians, paediatric and congenital cardiologists, cardiac surgeons, and anaesthesiologists and with other allied professions such as physiotherapists and dieticians.
All of these professionals in the multidisciplinary team can also provide psychosocial support; however, the disciplines that require professional education and training to specifically provide psychosocial care are as follows:

Child-life specialists: have a Bachelor's degree with course work in child development, child and family psychology, and early childhood education. They offer play experiences during hospitalisation, provide psychological preparation for medical procedures and healthcare-related events by giving age-attuned appropriate information and aftercare, and establish therapeutic relationships with children and parents to support family involvement in each child's care. ${ }^{18}$ Key elements in their approach are anxiety reduction, developmental stimulation, and offering distraction or a programme with daily activities during hospital stays. Child-life specialists working on the hospital ward have an important role in signalling psychosocial problems to other members of the team and in referring patients to other team members for psychosocial care if indicated. Counselling by a child-life specialist is related to the length of hospitalisation, and ends when the patient is transferred to another ward/hospital or goes home. (For an extensive review on preparing for medical procedures, we refer to the statement of the American Heart Association.) ${ }^{18}$

Medical social workers: should be key members of most cardiology units. ${ }^{18}$ Their role is diverse, encompassing family assessment, parental and family counselling, stress management, crisis intervention, psycho-education, and supporting families meeting 
tangible needs. From our perspective the main focus of social workers should be on counselling of parents and families on practical questions regarding work - for example, parental sick leave - finances and available community resources, transport to hospital, as well as on providing emotional support during hospitalisations. The frequency and intensity of the counselling depends upon the parental request for help. In case of transfer to another hospital or in the event of discharge, the medical social worker investigates the need for psychosocial care, and, if necessary, he/she arranges referral to a social worker and/or a psychologist operating in the region to which the patient has moved.

Religious counsellors: should be available to patients and families who desire religion-based counselling for example, praying and performing rituals. Counselling is provided when indicated or when requested by the family and is attuned to the specific cultural background of the patients and families.

Psychologists: usually have a university degree and when working in hospital environments they are most likely to also be specialised in psychotherapy. ${ }^{18}$

Help from a psychologist is needed in case of medically related trauma, behavioural or emotional problems - for example, anxiety of fear, depression, or anger - persistent adjustment problems following traumatic medical experiences - for example, anxiety, mood problems, tantrums, and medical noncompliance - inadequate coping mechanisms, mediation therapy, and end-of-life decisions. Psychological expertise is specifically needed for children who were already offered interventions in order to improve the child's life but in whom these interventions were not efficacious. Parental counselling by a psychologist, and similarly parental counselling provided by the medical social worker, may be indicated for problems associated with parenting the CHD child - for example, setting limits, stimulating autonomy in the child, reducing over-protectiveness. The frequency and duration of the sessions are established by taking into consideration the needs, treatment goals, and progress observed in the patients and their families.

Upon transfer to another hospital or at discharge, the psychologist discusses with the patient and the family whether there is still a need for psychological treatment, and, if so, where the psychological treatment can take place. If a family lives at considerable distance from the paediatric cardiology unit, referral to a local psychologist is discussed. Psychological treatment by an e-health portal can be an alternative, but adequate privacy should be ensured.

In case of parental psychopathology - that is, pre-existing parental psychopathology, not related to the child's condition - the psychologist discusses and, if necessary, arranges a referral to a psychologist/ psychiatrist or a mental health institution in the patient's local area.

Specialised licensed neuropsychologists: can perform neuropsychological examinations and provide psychotherapeutic treatment during hospitalisations or during outpatient contacts. Considering the fact that neurocognitive anomalies, although subtle, are common in CHD children, ${ }^{4,19,20}$ we recommend that such examinations be integrated into standardised care for CHD children to enhance their academic prospects and optimise their quality of life.

Child and adolescent psychiatrists: have a medical university degree (MD) and preferably specialised psychotherapeutic training. Most of the paediatric cardiology centres do not have child and adolescent psychiatrists, but they can be consulted on a referral basis. Expertise from child and adolescent psychiatrists is especially required when the problems are suspected to be psychiatric in nature and/or are persisting problems. These psychiatrists should be consulted in the following cases:

- psychotic hallucinations or delusions due to, for example, anaesthesia;

- psychiatric (co-)morbidity - such as Attention Defecit and Hyperactivity Disorders (ADHD), serious depression, anxiety, eating disorders suicidal ideation, threat of suicide, or when psychopharmaceutic medications such as antidepressants (Selective serotonin reuptake inhibitors SSRIs), tranquilisers, medication for ADHD, or antipsychotic medication is needed;

- during end-of-life decisions.

\section{Specialised nurses: paediatric nurse practitioners and advanced practice nurses}

Paediatric nurses or nurse practitioners: usually provide pre-procedural preparation during hospitalisation. Paediatric nurses focus on providing procedural preparatory information for children during hospitalisation, but they can also provide these services at outpatient clinics. ${ }^{18}$

Advanced practice nurses: also provide pre-procedural preparation for invasive procedures. They usually have knowledge of pathophysiology, child psychology, and child/family adjustments to chronic illness. Advanced practice nurses focus on disease management, psychosocial adjustment of child and family, and continuity of care. They can help the child and family in gaining an understanding of the cardiac defect, the necessary invasive procedures, and anticipated recovery and potential restrictions for example, regarding activities, school absence or offer anticipatory advice - for example, with regard to nutrition, children's reactions to procedures, etc. ${ }^{18}$ 


\section{Modules for multidisciplinary family-centred psychosocial care}

A referral to a professional from the psychosocial care multidisciplinary team should be made in consultation with a paediatric/congenital cardiologist or general paediatrician. Ideally, patients are discussed in a multidisciplinary team that includes medical workers, child-life specialists, religious and social workers, and psychologists. During regular meetings, the team assesses the needs of the family and decides what sort of help should be offered and by which member of the team.

Multidisciplinary family-centred psychosocial care can be offered to patients of various ages and in different situations. The specific modules are described below and depicted in Figure 1.

\section{Module A: antenatal care of parents}

Indication for psychosocial care: evaluation of stress and resilience within a family. The decision and referral for specialist parental guidance can be made on the basis of this evaluation. Fetal nurse specialists, when available in Fetal Cardiology Clinics, provide pertinent medical knowledge as well as support to families during the post-diagnostic phase.

Tools: assessment of psychosocial needs by a fetal nurse specialist, social worker, or a psychologist.

\section{Module B: psychological and neurodevelopmental follow-up}

Conforming to the guidelines from the American Heart Association and American Academy of Pediatrics we recommend ongoing neurodevelopmental assessments for children with CHD, especially when they are being followed up primarily as outpatients. ${ }^{21}$ It is vital that a close collaboration be established between paediatricians who are involved in the overall care of the patient.

Indication: for all children aged 9, 18, and 30 months and 5, 11, and 15/16 years.

Tools: screening by child/developmental and/or neuropsychologists using internationally well known and standardised questionnaires and test instruments with sound psychometric properties and adequate normative data from the general population.

For the specific questionnaires to be administered, we refer to the review by Marino et al. ${ }^{4}$ The questionnaires specified there address the following topics:

- neuropsychological functioning, including school grades and achievements;

- social, behaviourallemotional functioning;

- self-esteem;

- disease-specific quality of life.
If the screening process indicates an elevated level of psychosocial problems or the parents or patient expresses a need for psychosocial care, a referral can be made. The referral for psychosocial care can be aimed at extensive (neuro)psychological investigation, psychological/psychiatric treatment of the individual child, the parent-child relationship, or family treatment. In addition, parental guidance by a specialised health professional can be indicated.

\section{Module C: outpatient examinations}

Indication: when there are problems relating to the development and emotional/ psychosocial functioning of the child, parenthood and parenting the CHD child, and practical problems related to CHD, as reported by the parents, the patients, or the treating physician.

Examples of such problems are issues with acceptance and working through the illness, medical adherence, medical anxieties such as fear of needles, mood symptoms, aggression regulation - for example, outbursts of anger and tantrums - problems with eating or sleeping, existential issues, and problems pertaining to parenting or parenthood.

Tools: psycho-education, anxiety reduction, relaxation exercises, play therapy, cognitive behavioural therapy, eye movement desensitization and reprocessing and trauma-focused cognitive behavioural therapy, psychodynamic psychotherapy, specialist parent counselling, parent-child treatment, family therapy, mediation therapy, group psychotherapy, and psychopharmaceutic medication therapy.

These types of care are provided by the psychosocial team members.

\section{Module D: hospitalisations - cardiac catheterization, surgery}

Indication: when psychosocial care is required during hospitalisation. As stress levels are high for the family, especially for parents, prior to and during hospitalisation, ${ }^{22}$ the balance between the weight of emotional burden and the load capacity or resilience within a family should be evaluated at the start of hospitalisation. The aim of this evaluation is timely assessment of the psychomotor development of the child and the behavioural, emotional, and psychosocial functioning of the child and his or her family in order to arrange psychosocial care if necessary.

Patients and families who show psychosocial problems during this evaluation should be discussed in a meeting with the multidisciplinary team including the treating physicians to develop a counselling and treatment plan

Tools: stimulating psychomotor development of the child, anxiety reduction, relaxation techniques, 
game contacts, cognitive behavioural therapy, insight-oriented psychotherapy, specialist parental guidance, and mediation therapy by staff members of the psychosocial care team. Contact: at least once a week during hospitalisation.

\section{Module E: transition}

Transition service when available in an institution is provided by a team that is usually made up of a paediatric cardiologist, an adult congenital cardiologist, and the specialist nurse from the adult CHD service.

Indication: when problems occur during the transition from paediatric cardiology to the department of congenital adult cardiology

Tools:

- transition sessions with personnel from the medical team;

- leaflets about the transition and the congenital/ adult cardiology department;

- leaflets on young adult topics such as contraceptives, pregnancy, driver's licence, mortgages, health insurances, psycho-education days or workshops, and focus groups/group psychotherapy by staff members of the multidisciplinary psychosocial care team.

\section{Module F: death of a child}

Indication: when support is needed during the bereavement process and support in practical matters for parents and siblings.

Tools: anxiety reduction, relaxation exercises, play therapy, cognitive behavioural therapy, bereavement therapy, psychodynamic psychotherapy, specialist parent counselling, mediation therapy, and family therapy.

\section{Closure of psychosocial counselling or treatment}

A counselling and/or treatment trajectory is closed under the following conditions:

(a) the child is free from psychological problems;

(b) there is a marked decrease in psychosocial problems such that the family is able to lead a normal life;

(c) according to the child, the parents, and/or the practitioner of psychosocial care, the treatment trajectory can be closed; or

(d) if the child is discharged from the hospital and referred for long-term psychosocial care counselling or treatment in his or her area of residence.

\section{Conclusion and clinical implications}

Clinical experience and extensive research has shown that parents and children with CHD have specific requirements that necessitate psychosocial care. The guidelines for provision of such care stipulate the following:

- Multidisciplinary family-centred psychosocial care should be provided at both paediatric cardiology and adult congenital cardiology units. In order to achieve this goal, teams should include psychologists, social workers, and clinical nurse specialists who are specifically trained and dedicated to care for these patients and their families full time, and who can organise structural follow-up screening to identify psychosocial problems of children and parents in a timely manner.

- The needs of parents and children during the stressful period prior to cardiac surgery or invasive medical procedures should be identified in order to prepare the whole family for the procedure. If necessary, timely referral must be made to members of the psychosocial care team.

- Evidence-based, disease-specific, and familyoriented psychosocial interventions ${ }^{23}$ that are scientifically tested for efficacy should be further developed.

- Transition to the adult congenital cardiology service, guided by a member of the psychosocial care team, should be optimised.

- Interactive media, in the form of protected Internet information portal and/or apps providing medical and psychosocial information, with the possibility of interactive communication with psychosocial care providers and medical staff members for patients with CHD and their parents should be organised.

- It is important to be aware of the patient organisations in the local regions. This may help create a coordination of the psychosocial support both during hospitalisation and once the patients have been discharged. The collaboration with patient organisations could also be important to generate funding for psychosocial care when necessary.

Adequate psychosocial care can reduce the psychosocial problems of children and families, enhance the social, academic, and career prospects of these youngsters, and, not least important, may reduce noncompliance with medical treatment. Noncompliance may harm the patient's somatic health and result in related costs for medical care. ${ }^{24}$ In conclusion, in the longer term, adequate psychosocial care should be cost-effective, and, most importantly, should enhance the patients' and their families' quality of life.

\section{Acknowledgement}

None. 


\section{Financial Support}

This research received no specific grant from any funding agency, commercial or not-for-profit sectors.

\section{Conflicts of Interest}

None.

\section{References}

1. Spijkerboer AW, Helbing WA, Bogers AJJC, Van Domburg RT, Verhulst FC, Utens EMWJ. Long-term psychological distress, and styles of coping, in parents of children and adolescents who underwent invasive treatment for congenital cardiac disease. Cardiol Young 2007; 17: 638-645.

2. Bellinger DC, Newburger JW. Neuropsychological, psychosocial, and quality-of-life outcomes in children and adolescents with congenital heart disease. Prog Pediatr Cardiol 2010; 29: 87-92.

3. Karsdorp PA, Everaerd W, Kindt M, Mulder BJM. Psychological and cognitive functioning in children and adolescents with congenital heart disease: a meta-analysis. J Pediatr Psychol 2007; 32: 527-541.

4. Marino BS, Lipkin PH, Newburger JW, et al. Neurodevelopmental outcomes in children with congenital heart disease: evaluation and management a scientific statement from the American Heart Association. Circulation 2012; 126: 1143-1172.

5. Spijkerboer AW, Utens EMWJ, Bogers AJJC, Helbing WA, Verhulst FC. A historical comparison of long-term behavioral and emotional outcomes in children and adolescents after invasive treatment for congenital heart disease. J Pediatr Surg 2008; 43: 534-539.

6. Hofstra MB, van der Ende J, Verhulst FC. Child and adolescent problems predict DSM-IV disorders in adulthood: a 14-year followup of a Dutch epidemiological sample. J Am Acad Child Adolesc Psychiatry 2002; 41: 182-189.

7. Lesch W, Specht K, Lux A, Frey M, Utens E, Bauer U. Diseasespecific knowledge and information preferences of young patients with congenital heart disease. Cardiol Young 2014; 24: 321-330.

8. Levert EM, Helbing WA, Dulfer K, van Domburg RT, Utens EM. Psychosocial needs of children undergoing an invasive procedure for a CHD and their parents. Cardiol Young 2017; 27: 243-254.

9. McCusker CG, Doherty NN, Molloy B, et al. A controlled trial of early interventions to promote maternal adjustment and development in infants born with severe congenital heart disease. Child Care Health Dev 2010; 36: 110-117.

10. McCusker CG, Doherty NN, Molloy B, et al. A randomized controlled trial of interventions to promote adjustment in children with congenital heart disease entering school and their families. J Pediatr Psychol 2012; 37: 1089-1103.

11. Dulfer K, Duppen N, Kuipers IM, et al. Aerobic exercise influences quality of life of children and youngsters with congenital heart disease: a randomized controlled trial. J Adolesc Health 2014; 55: $65-72$.

12. Meentken MG, van Beynum IM, Legerstee JS, Helbing WA, Utens EM. Medically related post-traumatic stress in children and adolescents with congenital heart defects. Front Pediatr 2017; 5: 20 .

13. Walsh F. Traumatic loss and major disasters: strengthening family and community resilience. Family Process 2007; 46: 207-227.

14. Eiser C. Growing Up with a Chronic Disease: The Impact on Children and Their Families. Jessica Kingsley, London/ Philadelphia, 1993.

15. Craig FW, Lynch JJ, Quartner JL. The perception of available social support is related to reduced cardiovascular reactivity in phase II cardiac rehabilitation patients. Integr Physiol Behav Sci 2000; 35: 272-283.

16. Woolf-King SE, Anger A, Arnold EA, Weiss SJ, Teitel D. Mental health among parents of children with critical congenital heart defects: a systematic review. J Am Heart Assoc 2017; 6: pii: e004862. doi: 10.1161/JAHA.116.004862.

17. Logan DE, Simms S. Relationele benadering van crisis en conflict in de pediatrische kliniek. Gezinstherapie 2004: 373-391.

18. LeRoy S, Elixson EM, O’Brien P, Tong E, Turpin S, Uzark K. Recommendations for preparing children and adolescents for invasive cardiac procedures: a statement from the American Heart Association Pediatric Nursing Subcommittee of the Council on Cardiovascular Nursing in collaboration with the Council on Cardiovascular Diseases of the Young. Circulation 2003; 108: 2550-2564.

19. Snookes SH, Gunn JK, Eldridge BJ, et al. A systematic review of motor and cognitive outcomes after early surgery for congenital heart disease. Pediatrics 2010; 125: e818-e827.

20. Wernovsky G. Current insights regarding neurological and developmental abnormalities in children and young adults with complex congenital cardiac disease. Cardiol Young 2006; 16 (Suppl 1): 92-104.

21. Marino BS. New concepts in predicting, evaluating, and managing neurodevelopmental outcomes in children with congenital heart disease. Curr Opin Pediatr 2013; 25: 574-584.

22. Rahimianfar AA, Forouzannia SK, Sarebanhassanabadi M, et al. Anxiety determinants in mothers of children with congenital heart diseases undergoing cardiac surgery. Adv Biomed Res 2015; 4: 255.

23. Casey FA, Stewart M, McCusker CG, et al. Examination of the physical and psychosocial determinants of health behaviour in 4-5-year-old children with congenital cardiac disease. Cardiol Young 2010; 20: 532-537.

24. van der Wal MH, Jaarsma $\mathrm{T}$, Moser DK, Veeger NJ, van Gilst WH, van Veldhuisen DJ. Compliance in heart failure patients: the importance of knowledge and beliefs. Eur Heart J 2006; 27: 434-440. 